\title{
GIOVANNI ANTONIO PELLEGRINI: LA VIRTÙ INCORONATA DAL MERITO
}

\author{
Giorgio Fossaluzza
}

\author{
G. Fossaluzza \\ Università degli Studi di Verona \\ Dipartimento Culture e Civiltà \\ Via San Francesco, 22, 37129 Verona \\ giorgio.fossaluzza@univr.it
}

\begin{abstract}
L'inedito dipinto del veneziano Giovanni Antonio Pellegrini con La Virtù incoronata dal Merito è un'acquisizione ragguardevole per la fase avanzata del protagonista del Rococò europeo. Sono degni di nota la sofisticata eleganza compositiva, il virtuosismo dell'esecuzione, la leggerezza cromatica, l'espressività accattivante. L'invenzione allegorica attinge al repertorio di Cesare Ripa (1593; 1603), fonte primaria per artisti d'ogni tempo e d'ogni luogo. Ad esso guarda anche Giambattista Tiepolo che raffigura il Merito nelle decorazioni di due ville del vicentino: quella del conte Niccolò Loschi al Biron di Monteviale del 1734 e dell'avvocato Carlo Cordellina a Montechio Maggiore del 1743.
\end{abstract}

Parole chiave: Giovanni Antonio Pellegrini, Cesare Ripa, Giambattista Tiepolo

L'inedito dipinto (tela, 147x82 cm), già in collezione privata di Amburgo, per la qualità esecutiva è da ritenersi un'acquisizione importante nel catalogo della fase avanzata di Giovanni Antonio Pellegrini (Venezia 1675 -1741), circa il 1730. ${ }^{1}$ Esso possiede tutta la leggerezza del colore e la sofisticata eleganza delle forme e delle espressioni che fecero del maestro veneziano quale "pittore di storia" uno dei maggiori, dei più riconosciuti protagonisti del Rococò in Europa, precisamente in Inghilterra, come pure in Francia, Germania e Austria.

L'invenzione allegorica è quella che Pellegrini predilige. Con essa egli corrisponde al gusto di una civiltà raffinata che vuole esaltati i propri ideali in una dimensione immaginaria, serena o anche gioiosa che raramente ha contatti con la realtà. L'iconografia del dipinto si può svelare con precisione attingendo al più diffuso repertorio di Cesare Ripa (1593; 1603), fonte primaria per artisti d'ogni tempo e d'ogni luogo. ${ }^{2}$ Coprotagonista è la Virtù presentata come giovane bella e graziosa, in quanto ella mai invecchia, anzi prende vigore con il tempo, divenendo il maggiore ornamento dell'animo. È alata perché

\footnotetext{
${ }^{1}$ Ringrazio la Galleria Voena+Robilant di Milano e Londra per l'invito a studiare il dipinto che qui si pubblica. Lo si accompagna con l'elaborazione di un testo risalente al febbraio 2010, edito on line in lingua inglese.

Su Pellegrini basti il rinvio a George Knox, Antonio Pellegrini 1675-1741, Oxford, Clarendon press, 1995, pp. 126-127, 226, 244 cat. P. 249; Antonio Pellegrini: il maestro veneto del Rococò alle corti d'Europa, catalogo della mostra (Padova, Palazzo della Ragione, 20 settembre 1998 - 10 gennaio 1999), a cura di A. Bettagno, Venezia, Marsilio, 1998.

${ }^{2}$ Cesare Ripa, Iconologia overo descrittione dell'imagini universali cavate dall'antichità et da altri luoghi, Roma, eredi G. Gigliotti, 1593, pp. 167 (Merito), 289-291 (Virtù); Idem, Iconologia overo Descrittione di diverse imagini cavate dall'antichità, \& di propria inventione, trovate, e dichiarate da Cesare Ripa perugino (...) Di nuovo revista, \& dal medesimo ampliata di 400 el più imagini, et di figure d'intaglio adornata. Opera non meno utile che necessaria a poeti, Roma, L. Facio, 1603, pp. 313-315 (Merito), 506-512 (Virtù). Quest'ultima è la prima edizione illustrata con invenzioni del Cavalier d'Arpino. Nella presente circostanza si tiene conto della seguente edizione annotata, con particolare riguardo alle fonti: Cesare Ripa, Iconologia, a cura di Sonia Maffei; testo stabilito da Paolo Procaccioli, Torino, Einaudi, 2012, pp. 373-375, 595-601, 753-754, 842-844. Sul metodo di costruzione dell'Iconologia non si può tralasciare la menzione di Ernst H. Gombrich, Icones Symbolicae: The Visual Image in Neo-Platonic Thought, in "Journal of the Warburg and Courtauld Institutes», 11, 1948, pp. 163-192. Si vedano in seguito le osservazioni di Elizabeth Mc Grath, Personifying Ideals, recens. A G. Werner, Ripa's Iconologia. Quellen, Methode, Ziele, Utrecht 1977, in «Art History», VI, 3, 1983, pp. 363368; inoltre gli studi con particolare riguardo alle fonti erudite di Sonia Maffei, La politica di Proteo: trasformazioni e peripezie dell'Iconologia di Cesare Ripa, in Officine del Nuovo, Sodalizi tra letterati artisti ed editori nella cultura italiana
} 


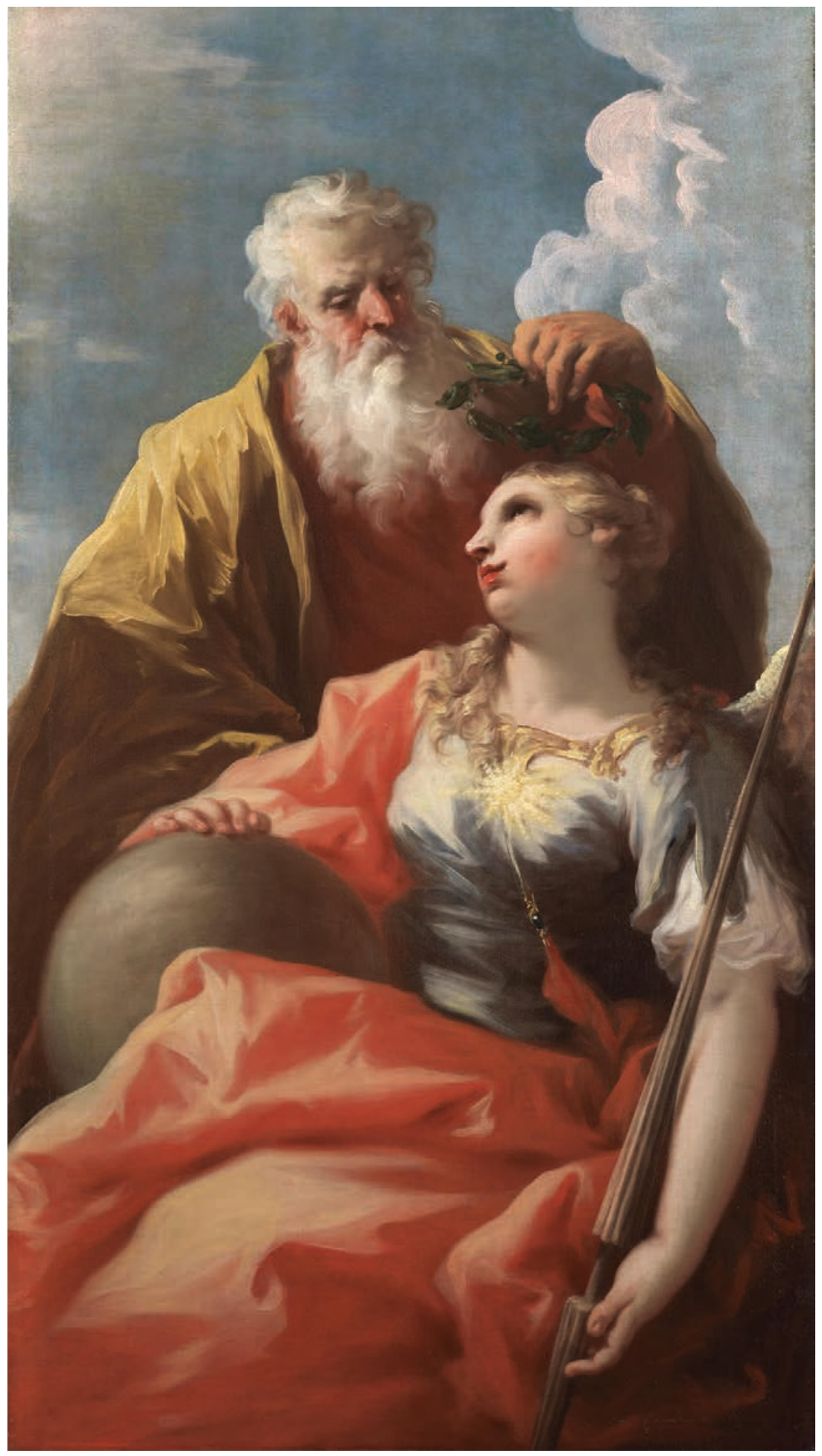

Fig. 1 - Giovanni Antonio Pellegrini, La Virtù incoronata dal Merito, collezione privata.

si alza sopra il senso comune; ha il sole nel petto perché, come l'astro illumina la terra, ella dal cuore dà vigore e scalda tutto il corpo dell'uomo. Regge l'asta perché alla Virtù è riconosciuto il governo, la

tra riforma e controriforma, atti del convegno internazionale (Utrecht, 8-10 novembre 2007), a cura di H. Hendrix e P. Procaccioli, Manziana, Vecchiarelli, 2008, pp. 479-495. Sulle caratteristiche delle edizioni e le varianti si veda Sonia Maffei, Per una concordanza diacronica dell' Iconologia di Cesare Ripa, in Repertori di parole e immagini. Esperienze cinquecentesche e moderni data bases, a cura di P. Barocchi e L. Bolzoni, Pisa, Scuola Normale Superiore, 1997, pp. 99-118. 
signoria ("maggioranza") sul mondo come significa la sfera che trattiene presso di sé, in deroga alle indicazioni di Ripa. ${ }^{3}$ Di solito la Virtù è coronata d'alloro in quanto esso è sempre verde e mai abbattuto dal fulmine, cioè dalle avversità. In questo caso è il vecchio canuto e dalla folta barba bianca, ammantato d'oro, a imporgli sul capo la ghirlanda della vittoria, della quale egli stesso normalmente si adorna. Si tratta infatti del Merito, come si può spiegare ricorrendo anche in tal caso a Ripa il quale attinge a Tommaso d'Aquino, in proposito richiamato in Polyantea nel 1592, così da qualificarlo come «azione virtuosa alla quale si deve qualche cosa pregiata in ricognizione». ${ }^{4} \mathrm{E}$ doveroso dunque offrire il giusto alla persona attiva, riconoscergli il "debito" che può essere di giustizia ("de condigno") nel caso di chi ha da ricevere, oppure di convenienza ("de congruo") nel caso di chi ha da dare il qualcosa. La prospettiva è quella delle opere buone compiute, da un lato, nell'amore di Dio e che pertanto sono frutto della grazia, dall'altro in funzione del puro merito di convenienza, nel qual caso esse giovano "O per conseguire beni temporali, o per disporsi alla grazia o per assuefarsi alla virtù di questa vita». ${ }^{5}$

Nell'attingere alla princeps dell'Iconologia si comprendono meglio, secondo quest'ultimo livello "temporale", i contenuti etico-morali che quest'allegoria esprime con Ripa e nella sua fortuna iconografica, compreso il legame con la Virtù: "Il Merito, essendo di per se stesso nobile, poco si cura della fortuna o di quei beni che da gli huomini si danno in ricompensa delle fatiche; però di esso si contenta nelle sue attioni il Magnanimo, il Forte, il Filosofo, il Sapiente et, come cosa inestimabile per eccellenza, credono che da se stesso si paghi, come l'oro, co'l quale è vestito, con l'oro medesimamente si compra. Quindi è che si dice la Virtù madre del Merito non havere fuor di se stessa guiderdone convenevole et non haver cosa che la possa pagare, come non ha cosa che la possa tener nascosta». ${ }^{6}$

L'identificazione iconografica e il valore attribuito a questa allegoria da Pellegrini sono accertati, in particolare, dal fatto che la Virtù e il Merito sono rappresentati anche in una delle tele che compongono l'importante ciclo che il pittore veneziano, lasciata l'Inghilterra, realizza fra il 1713 e il 1714 a Düsseldorf per il principe elettore palatino Giovanni Guglielmo II di Wittelsbach-Neuburg, conosciuto anche come Giovanni Guglielmo del Palatinato-Neuburg e detto Jan Wellem (Düsseldorf 1658-1716), da collocarsi nel castello di Bensberg (le superstiti ora a Monaco, Bayerische Staatsgemäldesammlungen, deposito al Castello di Schleissheim). ${ }^{7}$ In questo caso il Merito porge lo scettro alla Virtù che ha presso di sé l'aquila (anziché il globo). ${ }^{8}$ Da notare che la tela in pendant rappresenta La Virtù che incorona l'Onore che ha presso di sé la cornucopia, emblema quest'ultima di prosperità e anche di felicità pubblica. ${ }^{9}$ Altri

\footnotetext{
${ }^{3}$ Con questi attributi, ma priva della sfera è la rappresentazione della Virtù spettante a Pellegrini che appartiene a collezione privata di Vicenza, il cui taglio compositivo indica la probabile destinazione a un soffitto. Cfr. A. Bettagno, in Antonio Pellegrini 1998, pp. 216-217 cat. 61.

${ }^{4}$ Questi riferimenti sono precisati e analizzati da Maffei, in Ripa 2012, p. 753. Si tratta di Tommaso d'Aquino, Summa Theologiae, IIa-Ilae, q. 45, a.6; Domenico Nani Mirabelli, Polyanthea, hoc est, opus suavissimis floribus celebriorum sententiarum tam Graecarum, quam Latinarum exornatum, quos ex innumeris fere cum sacris, tum profanis auctoribus (...), Venezia, G. B. Ciotti, 1592, pp. 513-514.

${ }^{5}$ In generale si veda, ad esempio, Eugenio Valli, Il merito nella Summa Theologica di Tommaso d'Aquino, Friburgo 1990; quale voce sintetica quella in Giacomo Dal Sasso - Roberto Coggi, Compendio della Somma teologica di San Tommaso d'Aquino, Bologna, Studio domenicano, 1989, p. 449.

${ }^{6}$ Ripa 1593, p. 167.

${ }^{7}$ Sul ciclo che trae ispirazione da quello di Rubens per Maria de Medici da collocarsi nella Galleria del Palazzo di Lussemburgo si veda Klara Garas, Le Plafond de la Banque Royale de Giovanni Antonio Pellegrini, in "Bulletin du Musée Hongrois des Beaux-Arts», 1962, pp. 75-93, in part. p. 88 nota 25; Klara Garas, Giovanni Antonio Pellegrini in Deutschland, in Studi di storia dell'arte in onore di Antonio Morassi, Venezia, Alfieri, 1971, pp. 285-292; Matthias Reuss, in Bayerische Staatsgemäldesammlungen Alte Pinakothek, München. Venezianische Gemälde des 18. Jahrunderts. Vollständiger Katalog, bearb. von R. Kultzen und M. Reuss, München, Hirmer, 1991; Knox 1995, pp. 126-127, 226, 244 cat. P.249. ${ }^{8}$ Knox 1995, p. 121, fig. 101.

${ }^{9}$ Knox 1995, p. 121 fig. 100. Lo studioso (Knox 1995, pp. 121, 243 cat. P.241, 261 cat. P.445, fig. 102) ritiene facesse parte di questa decorazione il Nettuno (cm 174x80) sul mercato di Monaco di Baviera nel 1852, di recente segnalato in collezione Peron di Venezia, ben giudicabile nella riproduzione di Egidio Martini, Pittura veneta e altra italiana dal XV al XIX secolo, Rimini, Stefano Patacconi, 1992, pp. 292-293. Si devono ammettere tuttavia le forti affinità con l'opera qui presentata con datazione ben successiva.
} 
soggetti allegorici, assieme alla rappresentazione di divinità dell'Olimpo, costituivano un contorno alle scene memorative riguardanti il committente e la sua famiglia entro i riquadri del soffitto. Si tratta quindi di un doppio registro espressivo, l'uno storico anche contingente e l'altro allegorico, entrambi indirizzati alla medesima finalità celebrativa.

Quanto alla fortuna ravvicinata del tema in ambito veneto e a più complesse connessioni con altre allegorie, attinte ugualmente al repertorio di Ripa, è già stato proposto il richiamo a Giambattista Tiepolo, alla sua raffigurazione del Merito nelle decorazioni della villa del conte Niccolò Loschi al Biron di Monteviale del 1734 e della villa dell'avvocato Carlo Cordellina a Montecchio Maggiore del $1743 .{ }^{10}$ In questi complessi decorativi vicentini la Virtù non manca di accompagnarsi ad altre allegorie: nel primo affianca la Gloria e incorona l'Onore.

Il dipinto raffigurante La Virtù incoronata dal Merito di Pellegrini che qui si presenta per la prima volta in sede scientifica doveva avere una stessa funzione all'interno di un ciclo più vasto. Tale ipotesi è suggerita anche dal formato in verticale, quello adatto all'inserimento entro scomparti (probabilmente in stucco) da parete o soffitto che fungevano da contorno a riquadri di maggiori dimensioni e a più vasto sviluppo figurativo. La Sala d'Oro del Mauritshuis a L'Aja del 1718 ne è la più celebre esemplificazione, rara per integrità. ${ }^{11}$ La spazialità del dipinto qui illustrato - che è di illusorio respiro anche perché senza ancoraggi prospettici - sembra obbedire a una tale ipotizzata collocazione. Tuttavia, l'esame delle opere che figurano nel catalogo generale di Giovanni Antonio Pellegrini, compresi i disegni, il controllo delle altre che si sono aggiunte più di recente, non consente di proporre un diretto accostamento riguardo la tematica e il formato, in modo da definirne il contesto dell'originaria appartenenza. ${ }^{12}$ Le fonti dirette, poi, che riferiscano di decorazioni e cicli perduti sono per lo più parche di indicazioni precise che siano utili a tale proposito ricostruttivo; il caso di quella perduta o dispersa del castello di Bensberg ne è un'eccezione.

La qualità dell'opera che qui si presenta, come sopra osservato al riguardo della realizzazione pittorica e dell'invenzione, non lascia dubbi sul fatto che dovesse appartenere a un contesto di alto prestigio, tale da richiedere la dimostrazione del massimo impegno da parte del maestro. La composizione monumentale, la dilatazione delle forme possenti, la stesura cromatica magistralmente sciolta e tuttavia - occorre sottolinearlo - senza effetti flou spesso raggiunti da Pellegrini, semmai a larghe pennellate costruttive lasciate alla prima, sono tutti aspetti che offrono indicazioni confortanti per una collocazione cronologica. Essa, tuttavia, non è immediata a stabilirsi all'interno di un percorso che nella fase matura è di straordinaria coerenza stilistica. Con qualche anticipo rispetto a quanto prospettato in un primo tempo, si propone la realizzazione nell'ambito dell'attività che Pellegrini svolge tra Vienna, Venezia e Padova circa il 1730. Facendo riferimento alle opere di cronologia accertata, si pongono a monte la pala raffigurante Cristo consegna le chiavi a Pietro del 1727 della Salesianerinnenkirche e la decorazione distrutta con l'Assunzione della Vergine e Figure allegoriche della Schwarzspanierkirche di Vienna. ${ }^{13}$ Come nucleo di appoggio successivo si deve guardare al Cristo che risana il paralitico della Karlskirche di Vienna (1731-1734) il cui modelletto è al Museo di Belle Arti di Budapest, si aggiunga La Modestia presenta la Pittura all'Accademia che è morceau de reception all'Académie Royale de la Peinture di Parigi del 1733 (Parigi, Musée du Louvre); fa seguito il Martirio di santa Caterina, pala per la Basilica di Sant'Antonio di Padova del 1735 (ora al Museo Antoniano) che è di tutt'altra libertà esecutiva e pres-

\footnotetext{
${ }^{10}$ Per il primo caso Michael Levey (Giambattista Tiepolo. His Life and Art, New Haven-London, Yale University, 1986, pp. 68-70) osserva come si possa dimostrare che Tiepolo abbia tenuto conto delle varianti apportate all'edizione dell'Iconologia data alle stampe nel 1611. Per il rapporto fra il Merito e il Consiglio rappresentati in Villa Cordellina si veda Barbara Mazza, Il trionfo della ragione. Giambattista Tiepolo per Carlo Cordellina, in I Tiepolo e il Settecento vicentino, catalogo della mostra (Vicenza, Montecchio Maggiore, Bassano del Grappa, 26 maggio - 20 settembre 1990), a cura di F. Rigon et al., Milano, Electa, 1990 pp. 306- 320, in part. p. 314 cat. 2.5.

${ }^{11}$ Knox 1995, pp. 137-144, 237 catt. P.394-P.402.

12 Il catalogo più completo spetta a Knox 1995, pp. 225-277.

${ }^{13}$ Knox 1995, pp. 197-198, 263 cat. P. 486, fig. 162, 263 cat. P. 488, fig. 164.
} 
enta ancora effetti di cromatismo "svaporato" affine agli esiti di Rosalba Carriera. ${ }^{14}$ Si prosegue con le tele che Pellegrini realizza nel 1736 e 1737 su incarico di Karl Philipp von der Pfalz per la decorazione dei soffitti di quattro sale della Residenz di Mannheim (distrutte nel secondo conflitto mondiale) in cui si colgono ancora diretti riscontri stilistici, nei termini più specifici. ${ }^{15}$ L'accostamento è reso più convincente anche dalle affinità figurative. La Virtù trova un corrispettivo, ad esempio, in una delle Ore dell'ovale che raffigura l'Aurora già nella Roter Saal. ${ }^{16}$ Il Merito vede il suo corrispondente, non solo tipologico, nel Trionfo del Palatinato già nella Audienzzimmer. ${ }^{17}$

In questa fase, circa il 1730, trova altresì collocazione per ragioni stilistiche una serie di dipinti da stanza, come ovvio privi di certificazioni documentarie per un accertamento cronologico. ${ }^{18}$ Tra questi si possono selezionare per il riscontro comparativo, ad esempio, l'Allegoria della Pittura e l'Allegoria della Scultura provenienti dalla collezione Alvise Giustinian-Recanati (ora Venezia, Gallerie dell'Accademia, per acquisto del 1959). ${ }^{19}$ A una fase successiva, alla seconda metà degli anni Trenta, si ritiene possano appartenere invece i due ovali con Flora (Allegoria della Primavera) e Cerere (Allegoria dell'Estate) tuttora in collezione Giustinian-Recanati a Venezia. ${ }^{20}$ Possono situarsi in "un momento di passaggio", nei primi anni Trenta, la Venere e Cupido di collezione privata di Rovigo, il Bacco e Cerere già in collezione privata di Roma, le versioni dell'Allegoria della Primavera e dell'Allegoria dell'Estate, rispettivamente già in collezione Sonino a Venezia e sul mercato antiquariale viennese, inoltre l'Allegoria della Primavera segnalata in collezione privata di Palermo che va assieme all'Allegoria dell'Innocenza in collezione privata veneziana, le quali si trovavano in origine in Palazzo Salom. ${ }^{21}$ Rispetto a

\footnotetext{
${ }^{14}$ Knox 1995, pp. 200-205, 228 cat. P.31, 250 cat. P.332, 251 cat. P.342, 263 cat. P.484, figg. 166-169.

${ }_{15}^{15}$ Hans Huth et al., Die Kunstdenkmäler des Stadtkreises Mannheim, München, Deutscher Kunstverlag GmbH, 1982; Knox 1995, p. 241, catt. P.204-P.207, figg. 171-178.
}

${ }^{16}$ Huth 1982, pp. 278-282; Knox 1995, fig. 171.

${ }^{17}$ Huth 1982, pp. 285-287; Knox 1995, fig. 177. Si tratta dell'ultimo grande impegno dell'artista che non conosce crisi ed è ancora nel 1737 impegnato a Würzburg, in opere per le chiese di Frankenthal e Füssgonheim presso Mannheim prima del rientro definitivo a Venezia. La realizzazione delle sovrapporte per la Residenz, già fissata in tale circostanza, è stata anticipata al 1722 da Knox 1995, pp. 216, 265 catt. P.504-P.509, figg. 144-147. La decorazione della Stadtpfarrkirche di Frankenthal è stata distrutta nel 1943, ma è documentata da fotografie d'archivio. Cfr. Knox 1995, pp. 210-216, 232 cat. P.85, 233 cat. P.89, figg. 181, 182.

${ }^{18}$ Con riferimento ai principali cataloghi utilizzati, la selezione avviene in presenza di opere la cui attribuzione a Pellegrini è da respingere, per quanto si giudichino solo in riproduzione. Cfr. Egidio Martini, La pittura veneziana del Settecento, Venezia, Edizioni Marciane, 1964, p. 29, tav. 31: Zefiro, Venezia, collezione privata; tav. 32: Morte di san Francesco Saverio, Venezia, collezione privata (Mariotti?); p. 164 nota 70, fig. 71: Tobia e l'angelo, Princeton, Collezione Cannon; Idem, La pittura del Settecento Veneto, Maniago, Istituto per l'Enciclopedia del Friuli Venezia Giulia, 1982, p. 481 nota 72, fig. 423: San Pietro e l'angelo, Londra, collezione privata; p. 481 nota 69, fig. 424: Sofonisba, già Venezia, collezione privata; p. 482 nota 78, fig. 428: Agar nel deserto, già Londra, collezione privata. Si veda inoltre Adriano Mariuz, Antonio Pellegrini a Padova, in Antonio Pellegrini 1998, p. 34: La testa di san Giovanni Battista presentata a san Marco, Vescovana, chiesa parrocchiale, no Pellegrini ma Migliori o Mariotti; Bettagno, in Antonio Pellegrini 1998, pp. 140-141 cat. 17, 144-145 cat. 19, di dubbia autografia, forse della fase finale; 148-149 cat. 22; 200-201 cat. 51: opera dubbia. ${ }^{19}$ Knox 1995, p. 258 catt. P.410-P.411; Bettagno, in Antonio Pellegrini 1998, pp. 210-212, catt. 57-58: estate 1728. Si propongono a confronto i dipinti raffiguranti Flora di collezione privata torinese e Imene e Cupido in collezione Contarini a Venezia. Cfr. Martini 1964, pp. 164 nota 68, 173 nota 70, figg. 58, 70.

${ }^{20}$ Per i dipinti Giustinian-Recanati cfr. Knox 1995, p. 260 catt. P.441-P.442; Bettagno, in Antonio Pellegrini 1998, pp. 189-191 catt. 45-46: con datazione ai primi anni Venti. In quest'ultimo arco temporale dell'estrema attività di Pellegrini a Venezia si collocano nella presente occasione, inoltre, l'Erminia tra i pastori già Ball und Graüpe a Vienna, ora in collezione privata di Venezia, cfr. Martini 1992, p. 290 fig. 213; Bettagno, in Antonio Pellegrini 1998, pp. 198-199 cat. 50: 1730 circa; Cristo e la Samaritana, già Londra, M.R. Waddingham, poi Venezia, collezione privata, cfr. Bettagno, in Antonio Pellegrini 1998, pp. 192-193 cat. 47. Si aggiunga la Maddalena, già Venezia, collezione Rizzoli edita da Martini 1964, p. 173 nota 70, fig. 65.

${ }^{21}$ Sul dipinto di Rovigo si veda Martini 1982, p. 20, fig. 58: ultimo periodo veneziano; per il dipinto romano si rinvia a Martini 1964, p. 164 nota 68, tav. 42. Le allegorie già Sonino e a Vienna (casa d'aste Dorotheum) sono segnalate e 
questi esempi, un senso più costruttivo della forma, ma in presenza di un fondo scuro, è raggiunto in modo del tutto analogo nella Santa Caterina appartenuta al maresciallo von Schulenburg di Hannover (ora collezione privata), in cui l'effetto quasi di "non finito" per disinvoltura nella conduzione pittorica si ritiene sia quello proprio di un abilissimo e consumato frescante quale fu Pellegrini. ${ }^{22}$ Per quanto riguarda i suoi dipinti di destinazione devozionale pubblica si seleziona, inoltre, per il riscontro stilistico la lunetta della Sacra Famiglia, santa Elisabetta e san Giovanino dell'Oratorio dell'Istituto delle Nobili Dimesse di Padova. ${ }^{23}$

Pertanto, il dipinto della Virtù incoronata dal Merito che qui si illustra, proveniente da un contesto decorativo di vasto respiro, trova giustificazione della sua collocazione stilistica circa il 1730 soprattutto attraverso il confronto con le opere da stanza che si sono distribuite nell'ultimo decennio d'attività. Destinate a collezionisti dal gusto sofisticato e alla moda dovettero contribuire grandemente alla fortuna professionale di Pellegrini, la quale gli arrise anche nell'ultima fase, sempre operativa ma alleviata dagli affanni della mobilità internazionale che lo impegnò per tutta la vita e solo in fine trovò sosta. Lo assicura Guarienti nel 1753, nella biografia aggiunta a quelle di Orlandi: «Dopo varij e lunghi viagi per la Germania, Francia, ed Inghilterra, nei quai paesi operando non poche faccoltà raccolse, fissò sua stanza in Venezia, del premio di sue fatiche comodamente ed onestamente vivendo». ${ }^{24}$

riprodotte da Martini 1982, p. 482 nota 78, fig. 59; Idem 1992, p. 288 figg. 210-212. Per il dipinto segnalato a Palermo cfr. Martini 1964, p. 32, tav. 46; Idem 1992, pp. 288-289 cat. 125: con datazione tra il 1737 e il 1741. L'Allegoria dell'Innocenza è edita da Martini 1964, p. 32, tav. 47. Completa il gruppo il dipinto di Venere e Amore segnalato a Verona, collezione Rodino da Martini 1964, p. 164 nota 68, tav. 37

22 Knox 1995, pp. 205, 241 cat. P. 200, fig. 170, tav. XIII; Bettagno, in Antonio Pellegrini 1998, pp. 212-213 cat. 59.

${ }^{23}$ Per la datazione e le fonti storiografiche basti il rinvio al contributo di A. Mariuz 1998, pp. 30-32: circa 1732. Si veda inoltre Bettagno, in Antonio Pellegrini 1998, pp. 208-209 cat. 56.

${ }^{24}$ Cfr. Pellegrino Antonio Orlandi, Abecedario pittorico (...) contenente le notizie de' Professori di pittura, scultura ed architettura e nobilmente di nuove notizie accresciuto da Pietro Guarienti, Venezia, G. Pasquali, 1753, p. 79. 


\section{GIOVANNI ANTONIO PELLEGRINI: VRLINU KRUNI ZASLUGA}

Neobjavljena slika Venecijanca Giovannija Antonija Pellegrinija „Vrlinu kruni Zasluga“ važna je akvizicija iz posljednje faze jednog od protagonista europskog rokokoa. Vrijedni su pažnje kompozicijska elegancija, virtuoznost izvedbe, kromatska lakoća te nježna ekspresivnost. Alegorijska inventivnost nastavlja se na repertoar Cesarea Ripe $(1593 ; 1603)$ kao primarnog izvora za umjetnike svih vremena i mjesta. Ovime se inspirira i Giambattista Tiepolo koji prikazuje Zaslugu na dekoracijama dviju vila na vićentinskom području: u vili grofa Niccoloa Loschija u Biron di Monteviale iz 1734. i onoj odvjetnika Carla Cordellina u Montecchio Maggiore iz 1743.

Uzimajući u obzir Ripine tekstove, Pellegrinijeva alegorija manifestira dubinu njegova morala i etike. Glavni lik je Vrlina prikazana kao mlada graciozna djevojka koja ne stari, već vremenom osnažuje i postaje najvećim ukrasom duše. Sijedi starac s gustom, sijedom bradom, zaogrnut zlatom, stavlja joj na glavu vijenac pobjede kojim se inače on sam krasi. Riječ je o Zasluzi koja nudi dostojnu nagradu osobi koja je to svojim djelovanjem zaslužila. Perspektiva za promišljanje je ona od dobrih djela napravljenih, s jedne strane iz ljubavi prema Bogu koja su plod milosti, a s druge strane imaju i funkcionalu ulogu te su napravljena čistom zaslugom praktičnosti, u kojem slučaju su, prema Tomi Akvinskom od kojeg polazi Ripa, korisna „ili da bi se dostigla vremenska dobra, ili da bi se raspolagalo milošću ili naviklo na vrline u ovom životu."

Slika proizlazi iz dekorativnog konteksta velikih razmjera, jer se trebala pridružiti drugim slikama na stropu ili zidu koje su razvijale ikonografski program složenog značenja. Datacija oko 1730-e utemeljena je ponajviše usporedbom sa sličnim djelima „da camera" iz zadnjeg desetljeća djelovanja. Tražene od mnogih kolekcionara sofisticiranog ukusa i mode, pridonijele su Pelleginijevom dobrom statusu u posljednjim godinama. Nakon radova u Beču i mnogih po Njemačkoj, on se povlači i radi u Veneciji gdje "kao nagradu za svoj rad živi udobno i iskreno" (Orlandi, 1753).

Ključne riječi: Giovanni Antonio Pellegrini, Cesare Ripa, Giambattista Tiepolo

Prevela: Marija Bijelić 